\title{
Preparing a trainee for the MRCPsych examinations
}

\author{
Richard Mindham
}

Since the formation of the College in 1971 and the introduction of the examination for membership of the College in 1973, preparation of candidates for the examination has been subject to a wide range of influences which have shaped all aspects of psychiatric training. Possibly the most important factor has been the consensus that membership of the College should mark the end of general training and the beginning of specialist training in psychiatry. This is in contrast to some similar diplomas which may be required as an entry qualification to postgraduate medical training (e.g. MRCP) or others which may mark the end of specialist training and be awarded only shortly before achieving consultant status (e.g. MRCPath).

Falling in the middle of postgraduate training in this way means that membership of the College is neither a filter to remove candidates unfitted for a career in psychiatry nor a certificate of competence to undertake independent professional practice. The introduction of the Part I examination for MRCPsych, which includes a clinical examination, taken after a minimum of one year of training, was intended to act as something of a filter but it is unclear whether it does exclude unsuitable candidates from further training and from membership of the College.

Only after becoming a Member of the College and obtaining a Specialist Registrar (Senior Registrar) post does a trainee commence training in the psychiatric specialities and subspecialities. This means that all psychiatrists have a common training before specialisation. This contrasts with the arrangements in some other countries; in the USA for example, training in child and adolescent psychiatry is quite separate and is likely to include little experience in other areas. The pattern we have adopted greatly influences the content of training and of the examination. Preparation for the examinations for the MRCPsych must prepare candidates for training in the psychiatric specialities and ensure that they have sufficient knowledge of the specialities they are discarding to allow them to pursue their future work. Thus, the main objective of psychiatric training leading to the MRCPsych examination is to provide a generic grounding in psychiatry which will fit candidates for further training in any of the psychiatric specialities: general psychiatry, the psychiatry of old age, forensic psychiatry, child and adolescent psychiatry, the psychiatry of learning disability, and psychotherapy; and in the subspecialities of general psychiatry which include: liaison psychiatry, drug dependence and rehabilitation.

\section{The process of training}

For many years it has been recognised that in undergraduate medicine there is a tension between medical education and training in the skills of a competent practitioner (Allbutt, 1906; Flexner, 1910; General Medical Council, 1993). The need for clinical competence at the time of completion of specialist training is not controversial in itself, but the validity of the methods used in assessing competence is. More difficult is to prepare a trainee not only to practice competently but to be able to assimilate new methods of management, new approaches to the organisation of services and new technologies. This requires a capacity to evaluate new methods and to use them appropriately alongside what already exists. Psychiatric training has tried to accommodate both of these needs: clinical competence with a capacity for development. Perhaps the process of reaching this objective

R. H. S. Mindham, FRCPsych, is Nuffield Professor of Psychiatry at The University of Leeds, Division of Psychiatry and Behavioural Sciences in Relation to Medicine, 15 Hyde Terrace, Leeds LS2 9LT. He was Chairman of the Joint Committee on Higher Psychiatric Training and is now Chief Examiner of the College and Dean of the Faculty of Medicine, Dentistry and Health in the University of Leeds. 
is to acquire current knowledge of our subject but in an historical context. This requires an understanding of how we have reached our current state of knowledge, which in turn gives pointers as to how new information will be incorporated into practice in the future. In all clinical subjects there is a need to develop clinical competence, but be able to relate clinical practice to theoretical knowledge; psychiatry is no different.

These objectives face the trainee with a series of major tasks. Clinical competence requires clinical experience in a setting in which there is reflection on the trainee's work from experienced colleagues. The psychiatric literature is vast; the trainee needs guidance in sampling it. Reading is often from textbooks but should also allow the trainee to understand how knowledge has formed, the strengths and weaknesses in the evidence and the need for further investigation and evaluation of things which may seem at first sight to be established. This is the process of higher education which is well established in our universities: the student learns something of received wisdom; chooses topics for detailed examination from original sources of information; and undertakes a dissertation on a single topic, often with original data. Much of the information considered may well lose currency but the process learned is applicable to the evaluation of new knowledge.

Medical students become doctors able to carry out clinical evaluations of patients, to institute schemes of management under supervision, and to relate as a professional to patients, relatives and staff. Similarly, in postgraduate training in psychiatry the trainee passes through the process of becoming a psychiatrist, acquiring the skills, responsibilities and relationships that the role requires.

\section{Specific aims of training}

Trainees are required to have knowledge of the sciences thought to be basic to psychiatry, which include psychology, sociology, biochemistry, neuroanatomy, neurophysiology, neuropharmacology, epidemiology, research methodology and statistics. Study of the literature should include psychopathology and something of the psychiatric specialities. The curriculum has been outlined by the College (available from the College upon request). The greatest problem in providing a curriculum is the detail and balance which should be given to each topic. Possibly the best guides to this are the time available for study and the fact

\section{Box 1. Aims of training \\ Theoretical knowledge \\ Basic sciences \\ Clinical sciences \\ Clinical skills and experience \\ Research \\ Knowledge of methodology \\ Capacity to review research papers critically}

that, at this stage, trainees are not preparing to be specialists.

Clinical methods must also be acquired. At the time of becoming a Member of the College every trainee is expected to be able to make an assessment and provide a programme of management for any kind of patient seen in any setting. The trainee will also be expected to deal with patients' relatives and to relate constructively to colleagues in the mental health disciplines. However, they would not be expected to practice at the level expected of a specialist. The clinical capabilities required are also outlined in the curriculum prepared by the College. The specific requirements of candidates for the examination are given in the 'Guidelines for Candidates and Examiners', distributed by the College (available upon request) (Box 1).

\section{The membership examination}

The examination for membership generally reflects the requirements of a trainee at this stage of training; that they are ready to pass to the next stage of training. It has to be acknowledged, however, that many of the skills required of a psychiatrist are difficult to evaluate in a formal examination and have to be taught as a part of the process of training rather than in preparation for an examination.

The examination itself is in two parts. Part I is taken after a minimum of one year's experience in general or old age psychiatry, of which six months must be in general psychiatry. It is in two parts; the multiple choice question (MCQ) paper (Box 2) and the clinical examination. The MCQ paper is in psychology, psychopathology, psychopharmacology and psychiatry. The questions are in the 'Newcastle' style with a statement in the 'stem' followed by five subsidiary statements, or 'branches'. The candidate may attempt or decline 
Box 2. MRCPsych examination: Part I

Multiple Choice Question Paper

A test of factual knowledge; equally divided between basic sciences and clinical topics

The Clinical Examination

A test of competence in the assessment of individual patients; requires skill in history taking, examination of the mental state, diagnosis and evaluation of aetiological factors

to attempt each branch. A correct answer earns one mark; no attempt earns no mark but an incorrect answer carries a penalty of a loss of one mark. It is essential for candidates to familiarise themselves with the structure and marking of the MCQs. The clinical section of the Part I examination seeks to examine basic competence in the assessment of adult patients; it does not require a knowledge of treatment, management and prognosis.

The Part II examinations are more extensive (Box 3). First, there are two MCQ papers which require factual knowledge from the whole of the basic sciences and clinical curricula. Second, there is an essay paper in which two essays, each of threequarters of an hour are chosen, one from a choice of two in Section A on general topics and the other from two questions in Section B, on psychiatric specialities. In these essays candidates are expected to display a knowledge of the topic, to have some familiarity with the relevant literature, to be able to discuss conflicting views and evidence and to present their answer in a satisfactory literary style. These skills are felt to have some bearing on the role of the consultant in presenting written material to other doctors, to lawyers, to learned bodies, to journals and to managers.

Until the spring of 1999 or thereabouts there will also be the Short Answer Question (SAQ) paper, which is largely a test of factual knowledge. This paper is being phased out because it is thought to reflect similar knowledge to that examined in the MCQ papers. Instead, there will be a 'critical review' paper, which is being developed at the time of writing. This will probably require the assessment of a paper which has been published, with questions on the review of the literature, the description of the method, the presentation of the results, the use of statistical tests, and the evaluation of the findings in relation to the literature. Some of the paper, such as the summary, is likely to be removed. The aim of this paper will be to test ability to review literature critically and familiarity with the methodology of research. The introduction of this new paper will introduce a new element to the Part II examination, testing competence in an area previously neglected, and will thereby stimulate teaching of these accomplishments.

The clinical examination for Part II is now in two parts: the 'individual patient assessment' (formerly the 'clinical examination') and 'patient management problems' (formerly the 'oral examination'). The former tests clinical competence in the assessment and management of clinical problems. Assessment is given relatively less emphasis than in the Part I clinical examination but a knowledge of management, treatment, prognosis and legal aspects is expected. The 'Guidance for Candidates and Examiners' distributed by the College outlines how the examination is conducted. The 'patient management problems' represent a considerable change from the former oral examination. The Examiners prepare a selection of clinical management problems, which they present verbally to the candidate, who is expected to discuss the issues presented by each 'vignette'. The candidate is expected to display a grasp of the problems presented, to recognise the difficulties and dangers which might arise, to suggest methods of investigation and management which are relevant and

Box 3. MRCPsych examination: Part II

Multiple Choice Questions

Covering the whole syllabus - a test of factual knowledge

Essay Paper

One essay from each of two sections, the first from two general topics, the second two topics from the specialities

Short Answer Question Paper

A test of factual knowledge - to be replaced by a Critical Review Paper

The Clinical Examination

Patient Management Problems - a series of vignettes

Individual Patient Assessment - a development of Part I examination with knowledge of management, treatment, prognosis and legal matters required in addition

This examination must be passed as a whole but not necessarily each part 
realistic and to be able to present evidence from the literature for the measures they have chosen to adopt. Generally, four distinct clinical problems will be presented to each candidate in the course of the examination.

Candidates must pass the clinical examination to pass the Part II examination as a whole, no matter how well they may have performed on the other papers. This requirement has not changed but candidates are only required to pass the clinical examination as a whole, and not necessarily in each part. This change has been made to release individual Examiners from the opprobrium of being solely responsible for a candidates' failure: now a marginal fail on either patient management problems or individual patient assessment can be compensated by a good mark in the other part of the examination.

A research option has been available in Part II of the examination for some years. A dissertation topic and protocol requires preliminary approval by the Court of Electors and must be carried out under the supervision of an acceptable supervisor. The dissertation is examined by two specially appointed Examiners. If the candidate satisfies the Examiners, he/she is excused from the essay paper in the Part II examination. The research option has been infrequently offered by candidates.

\section{The training needs of candidates}

What guidance do trainees require of their educational supervisor to enable them to satisfy the Examiners of the College? There are three main needs: for theoretical teaching; the acquisition of clinical competence; and having a knowledge of research (Box 4; Box 5).

Teachers should recognise that preparation for the MRCPsych examination requires attendance at a comprehensive course of theoretical instruction over the three-year period of training. Only this kind of course can help the candidate to master the very considerable body of material covered by the curriculum. Teaching of the basic sciences by people working in these fields, but with an understanding of the needs of psychiatrists, is essential if the candidates are to gain a grasp of the subjects themselves, rather than a summary version prepared for trainees by non-experts. The teaching of clinical topics is easier to arrange but it should be remembered that candidates need teaching that is stimulating, up-to-date and critical. They can read standard textbooks themselves. Those
Box 4. Training needs

Attendance at a three-year course of theoretical instruction

Supervised clinical work in a range of specialities

A knowledge of research methods and assessment of the literature

arranging courses of instruction should remember that the needs of trainees change, for example neurology no longer dominates the medical component of teaching for psychiatrists but is now accompanied, and even rivalled, by subjects such as endocrinology. Theoretical teaching often includes reading seminars, journal clubs, problembased learning, and other approaches which bring diversity to long-term courses. Regular attendance is vital and arrangements should be made for trainees to be relieved of clinical duties to permit them to attend day-release courses. As a corollary, candidates for the MRCPsych examination should be expected to attend regularly as it is part of the contract with their employer to do so, as well as being in their best professional interests. In my view, short intensive courses have a very limited place in preparing for the MRCPsych. The process of education and learning simply cannot be undertaken solely on this basis.

Clinical experience is generally gained by rotation through a range of clinical placements. Schemes of this kind are taken for granted now but are a relatively recent phenomenon on a widespread basis, having been actively promoted by the College since its inception. Suitable supervision by the trainee's consultants is crucial during clinical work. Supervision is in many ways the educational topic of the moment, having many facets, many styles, and making many demands upon both trainee and supervisor. Central to the relationship between trainee and supervisor is the opportunity to discuss clinical problems through discussion of new patients, review of existing patients and the discussion of clinical problems. Trainees must develop a method of evaluating the full range of clinical cases and in reaching working plans of management; here the consultant has a central role. Rotation through different posts will bring the trainee into different clinical fields and allow them to experience supervision by consultants with different views and orientations. The trainee has to pick his or her way through this experience, selecting good practice from teachers on the way. 
Box 5. The role of the Educational Supervisor

Guidance in acquiring theoretical knowledge

Attendance at a suitable course

Reading

Discussion of theoretical issues

Supervision of clinical work

Assessment of new cases

Guidance in care of out-patients

Review of in-patients

Development of clinical skills

Participation in case conferences

Training in interviewing

Training in research

Critical review of papers

Attendance at journal clubs

Training in information technology

Guidance in selecting research topics

Participation in Supervisor's research

Supervision of research

(Educational Supervisors will not generally be able to fulfil all of these roles)

The level of experience of research required in training is debated, but there is a broad consensus that every trainee should develop the capacity to evaluate published research critically. Training is already given in the form of journal clubs and reading seminars but is likely to become more formalised as the requirements of the new examination become more clear. There is less agreement as to whether personal experience of research is a required part of training for future consultants. In my view it is. I believe that it is only by carrying out research that the trainee can come to a full acquaintance with the process of research and have an awareness of the difficulties encountered in conducting the simplest project. The supervisor may be able to help trainees by offering supervision of work or participation in existing research where appropriate.

\section{The longer-term perspective}

All schemes of training and the assessments that go with them undergo a process of evolution. Even in the 25 years since the College was founded we have seen major changes in psychiatric practice, in the organisation of services and in the number and diversity of psychiatric specialities. The content of teaching and of examinations reflects these changes and will no doubt continue to change in response to many factors (Box 6).

In the examinations for the MRCPsych we have seen the recent introduction of curricula for the basic sciences and for clinical topics. The preparation of a syllabus for training was in accord with the proposals of a government departmental working party on postgraduate medical training (Department of Health, 1993). The curriculum for the MRCPsych will clearly require revision on a regular if infrequent basis.

Requirements of experience are likely to become more specific in such areas as psychotherapy training. Certification of specific supervised experience is a requirement of the Australasian examinations. The use of log-books is another way of specifying and recording particular experiences in training; this approach has been a feature of training in obstetrics and gynaecology for many years and is being evaluated for use in psychiatry by the College.

Supervision is a central feature of training in most professional areas. There are many concepts and definitions of supervision but it is essentially the creation of a relationship between trainer and trainee which allows a constructive but critical review of the professional practice of the trainee. The College stresses the importance of supervision in training and is encouraging the development of this essential skill by trainers. This topic is going to figure prominently in assessment of training, the requirements of educational supervisors, at conferences for tutors and in discussions among trainees.

The types of assessment used in examinations will gradually change. For example, the new paper which assesses the ability to critically examine research evidence will be introduced within the next few years. This will clearly extend the range

Box 6. The future - requirements of trainees will change and may include:

Examination of critical assessment of research

More closely specified clinical experience

The use of log-books of experience

More structured supervision

New forms of examination

Personal experience of the conduct of research 
of the examination. Whether, in due course, all candidates will be required to have personal experience of carrying out research, as is required in the preparation of a dissertation, is unclear. The research option has not been popular and there are major problems in organising this kind of work on anything other than a local basis. Many examinations have used closed-circuit television to present clinical problems to candidates. There is certainly potential in this approach now that most hospitals have television systems. The use of closed-circuit television would allow the same material to be presented on different sites. Any new methods which are introduced to the examination will be thoroughly evaluated first and notice given to all concerned several years ahead of their introduction.

\section{The role of the educational supervisor}

The role of the educational supervisor is not an easy one but it is a role which most clinicians enjoy. The contact with the trainee is sometimes trying, but is usually stimulating and informative. What better continuing professional development for the consultant can there be?

A particular problem is that in rotational schemes the supervisor is one of several to whom the trainee looks for guidance during a period of training extending over years. Each supervisor must address the needs of the trainee at his or her particular stage of training. As we all know, basic aspects of practice are the most important for the development of a sound approach to clinical work. The supervisor will need to take care not to negate earlier aspects of training. Differences of opinion between trainers are usually seen clearly by trainees and should be openly discussed. After all, in the longer term, the trainee will select and reject what they please from the wisdom of their teachers.

\section{References}

Albutt, T. C. (1906) On Professional Education With Special Reference to Medicine. London: Macmillan.

Department of Health (1993) Hospital Doctors: Training for the Future (the report of the working group on specialist medical training). London: Her Majesty's Stationery Office.

Flexner, A. (1910) A Medical Education in the United States and Canada. Bulletin No 4. New York: Carnegie Foundation.

General Medical Council (1993) Tomorrow's Doctors. London: GMC.

\section{Acknowledgements}

I am grateful to Dr David Owens for his comments on a draft and to Mrs R. Hanning for the preparation of the paper. 